\title{
Effect of Preservatives and Temperature on Physico-Chemical Properties and Economics of Mango (Mallika) Pulp
}

\author{
Manisha Jangir $^{1}$, Virendra Singh ${ }^{1}$, Shalini Pilania ${ }^{1}$, K.B. Shukla ${ }^{2}$ and L.N. Mahawer ${ }^{1}$ \\ ${ }^{1}$ Department of Horticulture, ${ }^{2}$ Department of Plant Physiology, RCA, MPUAT, Udaipur \\ (Rajasthan), India \\ *Corresponding
}

\begin{abstract}
A B S T R A C T
This study was carried out to evaluate the best preservation way for mango pulp preservation. For this the pulp of mango were preserved with thirteen different treatment combinations viz., pulp preserved with potassium meta-bisulphite $0.05 \%\left(\mathrm{~T}_{1}\right)$, pulp preserved with potassium meta-bisulphite $0.1 \%\left(\mathrm{~T}_{2}\right)$, pulp preserved with sodium benzoate $0.05 \%\left(\mathrm{~T}_{3}\right)$, pulp preserved with sodium benzoate $0.1 \%\left(\mathrm{~T}_{4}\right)$, pulp preserved with sodium meta-bisulphite $0.05 \%\left(\mathrm{~T}_{5}\right)$, pulp preserved with sodium meta bisulphite $0.1 \%\left(\mathrm{~T}_{6}\right)$, pulp preserved with potassium sorbate $0.05 \%\left(\mathrm{~T}_{7}\right)$, pulp preserved with potassium sorbate 0.1 $\%\left(\mathrm{~T}_{8}\right)$, pulp preserved with sodium benzoate + potassium sorbate $0.05 \%$ each $\left(\mathrm{T}_{9}\right)$, pulp preserved with potassium meta bisulphite + potassium sorbate $0.05 \%$ each $\left(\mathrm{T}_{10}\right)$, pulp preserved with sodium meta bisulphite + potassium sorbate $0.05 \%\left(\mathrm{~T}_{11}\right)$, refrigeration $4 \pm 2^{0} \mathrm{C}\left(\mathrm{T}_{12}\right)$, frozen storage $-20^{\circ} \mathrm{C}\left(\mathrm{T}_{13}\right)$, These treatment combinations were evaluated under completely randomized design with four replications. The results revealed that low temperature $\left(-20^{\circ} \mathrm{C}\right)$ storage was better with over all qualitative attributes viz., TSS, sugars (reducing and total), ascorbic acid and $\mathrm{pH}$ higher and acidity lower. Physico-chemical parameters of samples were observed at fortnight interval during storage (up to 90 days) and at end of storage acceptable pulp were used for different products (RTS, Squash and jam) preparation and analyzed its quality.
\end{abstract}

\section{Introduction}

Mango (Mangifera indica L.) is one of the most important fruit crops of India having socio-economic significance. It belongs to family Anacardiaceae and originated from South-East Asia. India is the second largest producer of fruits after China, with a production of 889.77 million tons from an area of 72.01 million hectare. Mango ranks second after banana with annual production 185.05 million tons from an area of 22.16 million hectare with an average productivity of 7.3 MT. (Saxena et al., 2015). Mango fruit is widely accepted by consumers throughout the world for its succulence, sweet taste and exotic flavor, being called "The King of fruits". The fruits are consumed fresh and largely used in the food industry for the production of canned fruits, jam and concentrated juice (Tharanathan et al., 2006). The mango fruit is popular in international market due to its excellent flavour, attractive fragrance, beautiful colour, delicious taste and nutritional properties (Sivakumar et al., 2011). In addition, it is good source of total soluble solids (18 to $22.8 \%$ ), total sugars $(17.20 \%)$, acidity $(0.12 \%)$, ascorbic acid 
ranged between 6.8 to $38.8 \mathrm{mg} 100 \mathrm{~g}^{-1}$, carotenoids $\left(16.83 \quad \mu \mathrm{g} \quad 100 \quad \mathrm{~g}^{-1} \quad\right.$ pulp$)$, polyphenolic compounds and antioxidant (Yahia and Ornelas-Paz, 2010). Thus, mango fruit can contribute significant amount of health protective bioactive compounds to the diet during summers.

Mango is a short seasoned fruit and being highly perishable does not withstand even in cold storage. Therefore, most of the fruit processing industries preserve mango pulp for the manufacture of mango products round the year. Mango pulp is not generally consumed directly rather used as fillings for pastries, jams, sauces, fruit juices and drinks (Hussain et al., 2003). However, in India, ripe mango fruits are used for the preparation of canned slices, canned pulp, nectar, squashes, ready to serve beverage, juice, jam and osmotically dehydrated pieces etc. (Ramteke et al., 1999). Generally, partially processed mango, as an industrial viable raw material available round the year is mango juice as a base for the production of nectar and other beverages. The mango pulp is highly perishable in nature due to high moisture content hence, pulp is having shortest shelf life. Therefore, the efforts have been made in the present investigation in order to improve the shelf life of mango pulp by using the various safe preservatives and with their optimum concentrations and temperature variations. Keeping this in view the present experiment was conducted.

\section{Materials and Methods}

The experiment was carried out in Post Harvest Lab, Department of Horticulture, Rajasthan College of Agriculture, MPUAT, Udaipur (Rajasthan) during May to October, 2016. Fully mature and ripe mango $c v$. Mallika fruits procured from Agriculture Research Station, Banswara (Rajasthan) were used for experimentation. After bringing the fruits from the farm diseased, damaged and off type fruits were discarded. Fresh and physiologically mature mango fruits were washed with tap water to remove dirt and dust particles adhering to the surface of fruits and crushed for obtaining pulp. For the preservation of mango pulp, after washing, peeling and coring the flesh was cut into small pieces with stainless steel knives and pulp was made by using electric blender. After addition of water in $2: 1$, pulp was homogenized and passed through fine mesh to get uniform textured pulp. Pulp was then pasteurized in a water bath at a temperature of $82 \pm 2^{0} \mathrm{C}$ for 30 minutes to reduce the microbial load.

Obtained pulp was preserved with thirteen different treatments namely, pulp preserved with potassium meta-bisulphite $0.05 \%\left(\mathrm{~T}_{1}\right)$, pulp preserved with potassium metabisulphite $0.1 \%\left(\mathrm{~T}_{2}\right)$, pulp preserved with sodium benzoate $0.05 \%\left(\mathrm{~T}_{3}\right)$, pulp preserved with sodium benzoate $0.1 \% \quad\left(\mathrm{~T}_{4}\right)$, pulp preserved with sodium meta-bisulphite $0.05 \%$ $\left(\mathrm{T}_{5}\right)$, pulp preserved with sodium meta bisulphite $0.1 \% \quad\left(\mathrm{~T}_{6}\right)$, pulp preserved with potassium sorbate $0.05 \%\left(\mathrm{~T}_{7}\right)$, pulp preserved with potassium sorbate $0.1 \%\left(\mathrm{~T}_{8}\right)$, pulp preserved with sodium benzoate + potassium sorbate $0.05 \%$ each $\left(\mathrm{T}_{9}\right)$, pulp preserved with potassium meta bi-sulphite + potassium sorbate $0.05 \%$ each $\left(\mathrm{T}_{10}\right)$, pulp preserved with sodium meta bi-sulphite + potassium sorbate $0.05 \%\left(\mathrm{~T}_{11}\right)$, refrigeration $4 \pm 2{ }^{0} \mathrm{C}$ $\left(\mathrm{T}_{12}\right)$, frozen storage $-20^{0} \mathrm{C}\left(\mathrm{T}_{13}\right)$ and replicated thrice. Physico-chemical parameters of samples were observed at 0,15 , $30,45,60,75,90$ days of storage. The TSS content of pulp was directly measured by the "Digital Refractometer" (Brix: 0.0 to $53.0 \%$ ) at $20^{\circ} \mathrm{C}$ temperature. Ascorbic acid by $2,6-$ dichlorophenol - indophenols dye method and acidity content of pulp was determined by diluting the known volume of pulp with distilled water and titrating the same against standard N/10 sodium hydroxide solution, 
using phenolphthalein as an indicator (A.O.A.C., 1995). Reducing sugars was measured by following "DNS Method" (Miller, 1959). Total Sugar was estimated by using "Anthrone Method" (Dubois et al., 1951). The $\mathrm{pH}$ of the pulp was directly measured on the $\mathrm{pH}$ meter. The data were analyzed by using Completely Randomized Design (Fisher, 1950).

\section{Results and Discussion}

\section{TSS and total sugar}

It is evident from the data (Tables 1 and 2) that TSS and total sugar content of stored mango pulp increased with the advancement of storage period in all the treatments. The maximum TSS and total sugar content were recorded in the frozen storage treatment $\mathrm{T}_{13}$ $\left(22.0^{\circ} \mathrm{B}\right.$ and $\left.15.69 \%\right)$ and minimum in $\mathrm{T}_{7}$ $\left(18.40^{\circ} \mathrm{B}\right.$ and $\left.14.98 \%\right)$ during the storage. The increment in TSS content of preserved mango pulp during storage was probably due to conversion of free polysaccharides (starch) into monosaccharide (Jain and Nema, 2007). TSS and total sugar content during storage have positive proportional trend (Akhtar et al., 2010) in mango pulp, Chandra and Gehlot, 2006 in bael pulp) and Yadav et al., 2017 in guava pulp.

\section{Acidity and pH}

The results indicate that the acidity of mango pulp increased and $\mathrm{pH}$ was decreased slightly with the advancement of storage period (Tables 3 and 4). The maximum acidity $(0.88 \%)$ was recorded in the treatment $\mathrm{T}_{7}$ (PS@ $0.05 \%)$ and minimum $(0.55 \%)$ was in the frozen storage treatment $\left(\mathrm{T}_{13}\right)$.

Table.1 Effect of different preservatives and temperatures on TSS of mango pulp during storage

\begin{tabular}{|c|c|c|c|c|c|c|c|}
\hline \multirow[t]{2}{*}{ Treatments } & \multicolumn{7}{|c|}{ Storage duration (days) } \\
\hline & $\mathbf{0}$ & 15 & 30 & 45 & 60 & 75 & 90 \\
\hline $\mathrm{T}_{1}(\mathrm{KMS} @ 0.05 \%)$ & 17.50 & 17.80 & 17.80 & 18.00 & 18.20 & 18.40 & 18.80 \\
\hline $\mathrm{T}_{2}(\mathrm{KMS} @ 0.1 \%)$ & 17.60 & 18.20 & 18.90 & 19.20 & 19.70 & 20.20 & 20.80 \\
\hline $\mathrm{T}_{3}(\mathrm{SB} @ 0.05 \%)$ & 17.30 & 17.80 & 18.40 & 18.60 & 18.80 & 19.00 & 19.20 \\
\hline $\mathrm{T}_{4}(\mathrm{SB} @ 0.1 \%)$ & 17.40 & 18.00 & 18.60 & 18.80 & 19.00 & 19.10 & 19.30 \\
\hline T 5 (SMS@ 0.05\%) & 17.30 & 17.50 & 17.60 & 17.80 & 18.10 & 18.40 & 18.80 \\
\hline $\mathrm{T}_{6}$ (SMS@0.1\%) & 17.30 & 17.60 & 17.80 & 18.10 & 18.60 & 19.10 & 19.30 \\
\hline $\mathrm{T}_{7}(\mathrm{PS} @ 0.05 \%)$ & 17.10 & 17.40 & 17.60 & 17.70 & 17.90 & 18.20 & 18.40 \\
\hline $\mathrm{T}_{8}$ (PS@0.1\%) & 17.20 & 17.30 & 17.50 & 17.80 & 18.00 & 18.40 & 18.70 \\
\hline $\mathrm{T}_{9}(\mathrm{SB}+\mathrm{PS} @ 0.05 \%$ each $)$ & 17.30 & 17.50 & 17.90 & 18.10 & 18.60 & 19.00 & 19.30 \\
\hline $\begin{array}{l}\mathrm{T}_{10}(\mathrm{KMS}+\mathrm{PS} @ 0.05 \% \\
\text { each) }\end{array}$ & 17.50 & 18.20 & 18.40 & 18.80 & 19.20 & 20.00 & 20.30 \\
\hline $\begin{array}{l}\mathrm{T}_{11}(\mathrm{SMS}+\mathrm{PS} @ 0.05 \% \\
\text { each) }\end{array}$ & 17.40 & 17.60 & 17.80 & 18.00 & 18.60 & 18.80 & 18.80 \\
\hline $\mathrm{T}_{12}$ (Refrigeration $\left(4 \pm 2{ }^{0} \mathrm{C}\right)$ & 17.50 & 18.20 & 18.80 & 19.30 & 19.80 & 20.30 & 20.90 \\
\hline $\mathrm{T}_{13}$ (Frozen storage -20 & & & & & & & \\
\hline SEm \pm & $\begin{array}{l}1 / .00 \\
0.37\end{array}$ & $\begin{array}{c}18.40 \\
0.39\end{array}$ & $\begin{array}{c}19.30 \\
0.60\end{array}$ & $\begin{array}{l}20.00 \\
0.42\end{array}$ & $\begin{array}{c}20.70 \\
0.42\end{array}$ & $\begin{array}{c}21.40 \\
0.34\end{array}$ & $\begin{array}{c}22.00 \\
0.43\end{array}$ \\
\hline C.D. $(\mathrm{P}=0.05)$ & NS & NS & NS & 1.21 & 1.21 & 0.99 & 1.26 \\
\hline
\end{tabular}


Int.J.Curr.Microbiol.App.Sci (2017) 6(7): 2431-2438

Table.2 Effect of different preservatives and temperatures on total sugar (\%) content of mango pulp during storage

\begin{tabular}{lccccccc}
\hline Treatments & \multicolumn{7}{c}{ Storage duration (days) } \\
\cline { 2 - 8 } & $\mathbf{0}$ & $\mathbf{1 5}$ & $\mathbf{3 0}$ & $\mathbf{4 5}$ & $\mathbf{6 0}$ & $\mathbf{7 5}$ & $\mathbf{9 0}$ \\
\hline $\mathrm{T}_{1}$ (KMS @ 0.05\%) & 13.05 & 13.39 & 13.90 & 14.28 & 14.29 & 15.01 & 15.09 \\
$\mathrm{~T}_{2}$ (KMS@ 0.1\%) & 13.13 & 13.61 & 14.08 & 14.43 & 14.53 & 15.17 & 15.36 \\
$\mathrm{~T}_{3}$ (SB @ 0.05\%) & 13.09 & 13.35 & 13.88 & 14.27 & 14.30 & 14.98 & 15.13 \\
$\mathrm{~T}_{4}$ (SB@ 0.1\%) & 13.11 & 13.58 & 14.03 & 14.39 & 14.49 & 15.12 & 15.31 \\
$\mathrm{~T}_{5}$ (SMS@ 0.05\%) & 13.13 & 13.35 & 13.83 & 14.23 & 14.31 & 14.95 & 15.14 \\
$\mathrm{~T}_{6}$ (SMS @ 0.1\%) & 13.11 & 13.38 & 13.86 & 14.24 & 14.33 & 14.98 & 15.21 \\
$\mathrm{~T}_{7}$ (PS@ 0.05\%) & 13.12 & 13.23 & 13.74 & 14.13 & 14.22 & 14.67 & 14.98 \\
$\mathrm{~T}_{8}$ (PS@ 0.1\%) & 13.16 & 13.28 & 13.79 & 14.16 & 14.26 & 14.71 & 15.07 \\
$\mathrm{~T}_{9}$ (SB+PS@ @.05\% each) & 13.09 & 13.48 & 14.01 & 14.29 & 14.36 & 15.07 & 15.17 \\
$\mathrm{~T}_{10}$ (KMS+PS@ 0.05\% each) & 13.13 & 13.54 & 14.01 & 14.31 & 14.38 & 15.09 & 15.24 \\
$\mathrm{~T}_{11}$ (SMS+PS 0.05\% each) & 13.12 & 13.45 & 13.99 & 14.28 & 14.32 & 15.04 & 15.11 \\
$\mathrm{~T}_{12}$ (Refrigeration $\left(4 \pm 2{ }^{0} \mathrm{C}\right.$ ) & 13.16 & 13.65 & 14.13 & 14.56 & 14.78 & 15.21 & 15.44 \\
$\mathrm{~T}_{13}$ (Frozen storage -20 $\left.{ }^{\circ} \mathrm{C}\right)$ & 13.16 & 13.78 & 14.16 & 14.68 & 14.99 & 15.34 & 15.69 \\
SEm \pm & 0.20 & 0.20 & 0.18 & 0.10 & 0.15 & 0.12 & 0.12 \\
C.D. (P=0.05) & NS & NS & NS & 0.30 & 0.43 & 0.35 & 0.36 \\
\hline
\end{tabular}

Table.3 Effect of different preservatives and temperatures on acidity (\%) content of mango pulp during storage

\begin{tabular}{lccccccc}
\hline Treatments & \multicolumn{7}{c}{ Storage duration (days) } \\
\cline { 2 - 8 } & $\mathbf{0}$ & $\mathbf{1 5}$ & $\mathbf{3 0}$ & $\mathbf{4 5}$ & $\mathbf{6 0}$ & $\mathbf{7 5}$ & $\mathbf{9 0}$ \\
\hline $\mathrm{T}_{1}$ (KMS@ 0.05\%) & 0.47 & 0.58 & 0.59 & 0.61 & 0.62 & 0.65 & 0.72 \\
$\mathrm{~T}_{2}$ (KMS@ 0.1\%) & 0.45 & 0.50 & 0.50 & 0.52 & 0.53 & 0.54 & 0.60 \\
$\mathrm{~T}_{3}$ (SB @ 0.05\%) & 0.46 & 0.54 & 0.56 & 0.61 & 0.62 & 0.72 & 0.80 \\
$\mathrm{~T}_{4}$ (SB@ 0.1\%) & 0.42 & 0.54 & 0.55 & 0.56 & 0.58 & 0.59 & 0.66 \\
$\mathrm{~T}_{5}$ (SMS@ 0.05\%) & 0.46 & 0.53 & 0.55 & 0.61 & 0.62 & 0.71 & 0.79 \\
$\mathrm{~T}_{6}$ (SMS@ 0.1\%) & 0.46 & 0.52 & 0.54 & 0.59 & 0.61 & 0.70 & 0.78 \\
$\mathrm{~T}_{7}$ (PS@ 0.05\%) & 0.45 & 0.59 & 0.62 & 0.64 & 0.69 & 0.79 & 0.88 \\
$\mathrm{~T}_{8}$ (PS@0.1\%) & 0.43 & 0.55 & 0.57 & 0.62 & 0.64 & 0.74 & 0.82 \\
$\mathrm{~T}_{9}$ (SB+PS@ 0.05\% each) & 0.46 & 0.54 & 0.56 & 0.59 & 0.62 & 0.67 & 0.73 \\
$\mathrm{~T}_{10}$ (KMS+PS@ 0.05\% each) & 0.47 & 0.56 & 0.58 & 0.60 & 0.61 & 0.62 & 0.68 \\
$\mathrm{~T}_{11}$ (SMS+PS@ 0.05\% each) & 0.47 & 0.57 & 0.59 & 0.60 & 0.60 & 0.63 & 0.70 \\
$\mathrm{~T}_{12}$ (Refrigeration $\left(4 \pm 2{ }^{\circ} \mathrm{C}\right.$ ) & 0.44 & 0.48 & 0.49 & 0.50 & 0.50 & 0.53 & 0.56 \\
$\mathrm{~T}_{13}$ (Frozen storage -20 ${ }^{\circ} \mathrm{C}$ ) & 0.45 & 0.47 & 0.48 & 0.49 & 0.50 & 0.50 & 0.55 \\
SEm \pm & 0.01 & 0.03 & 0.01 & 0.01 & 0.01 & 0.01 & 0.02 \\
C.D. (P=0.05) & $\mathrm{NS}$ & $\mathrm{NS}$ & 0.04 & 0.04 & 0.04 & 0.03 & 0.05 \\
\hline
\end{tabular}


Int.J.Curr.Microbiol.App.Sci (2017) 6(7): 2431-2438

Table.4 Effect of different preservatives and temperatures on $\mathrm{pH}$ content of mango pulp during storage

\begin{tabular}{|c|c|c|c|c|c|c|c|}
\hline \multirow[t]{2}{*}{ Treatments } & \multicolumn{7}{|c|}{ Storage duration (days) } \\
\hline & $\mathbf{0}$ & 15 & 30 & 45 & 60 & 75 & 90 \\
\hline $\mathrm{T}_{1}$ (KMS@ 0.05\%) & 4.22 & 4.11 & 3.85 & 3.69 & 3.39 & 3.16 & 2.91 \\
\hline $\mathrm{T}_{2}$ (KMS@ 0.1\%) & 4.23 & 4.17 & 3.86 & 3.71 & 3.46 & 3.17 & 2.99 \\
\hline $\mathrm{T}_{3}(\mathrm{SB} @ 0.05 \%)$ & 4.24 & 4.12 & 3.85 & 3.69 & 3.41 & 3.17 & 2.96 \\
\hline $\mathrm{T}_{4}(\mathrm{SB} @ 0.1 \%)$ & 4.21 & 4.16 & 3.87 & 3.72 & 3.45 & 3.18 & 2.97 \\
\hline T5 (SMS@ 0.05\%) & 4.23 & 4.11 & 3.87 & 3.63 & 3.39 & 3.19 & 2.92 \\
\hline $\mathrm{T}_{6}(\mathrm{SMS} @ 0.1 \%)$ & 4.24 & 4.15 & 3.89 & 3.64 & 3.41 & 3.22 & 2.94 \\
\hline $\mathrm{T}_{7}$ (PS@ 0.05\%) & 4.23 & 3.93 & 3.79 & 3.49 & 3.29 & 3.11 & 2.86 \\
\hline $\mathrm{T}_{8}(\mathrm{PS} @ 0.1 \%)$ & 4.22 & 3.94 & 3.81 & 3.51 & 3.32 & 3.23 & 2.89 \\
\hline $\mathrm{T}_{9}(\mathrm{SB}+\mathrm{PS} @ 0.05 \%$ each $)$ & 4.24 & 3.98 & 3.84 & 3.57 & 3.39 & 3.19 & 2.98 \\
\hline $\mathrm{T}_{10}(\mathrm{KMS}+\mathrm{PS} @ 0.05 \%$ each) & 4.25 & 4.10 & 3.87 & 3.63 & 3.46 & 3.25 & 2.96 \\
\hline $\mathrm{T}_{11}(\mathrm{SMS}+\mathrm{PS} @ 0.05 \%$ each$)$ & 4.22 & 4.04 & 3.84 & 3.59 & 3.42 & 3.21 & 2.91 \\
\hline $\mathrm{T}_{12}$ (Refrigeration $\left(4 \pm 2{ }^{\circ} \mathrm{C}\right)$ & 4.23 & 4.18 & 3.96 & 3.95 & 3.87 & 3.80 & 3.72 \\
\hline $\mathrm{T}_{13}$ (Frozen storage $-20^{\circ} \mathrm{C}$ ) & 4.21 & 4.18 & 4.11 & 4.05 & 4.01 & 3.91 & 3.85 \\
\hline SEm \pm & 0.06 & 0.07 & 0.06 & 0.04 & 0.04 & 0.03 & 0.02 \\
\hline C.D. $(\mathrm{P}=0.05)$ & NS & NS & NS & 0.12 & 0.11 & 0.09 & 0.06 \\
\hline
\end{tabular}

Table.5 Effect of different preservatives and temperatures on ascorbic acid (mg 100g-1 of pulp) content of mango pulp during storage

\begin{tabular}{|c|c|c|c|c|c|c|c|}
\hline \multirow[t]{2}{*}{ Treatments } & \multicolumn{7}{|c|}{ Storage duration (days) } \\
\hline & $\mathbf{0}$ & 15 & 30 & 45 & 60 & 75 & 90 \\
\hline $\mathrm{T}_{1}(\mathrm{KMS} @ 0.05 \%)$ & 25.02 & 23.54 & 22.01 & 19.01 & 17.02 & 14.99 & 12.56 \\
\hline $\mathrm{T}_{2}$ (KMS@ 0.1\%) & 25.03 & 23.67 & 22.17 & 19.12 & 17.34 & 15.12 & 12.76 \\
\hline $\mathrm{T}_{3}(\mathrm{SB} @ 0.05 \%)$ & 24.98 & 21.97 & 19.73 & 18.23 & 16.65 & 14.61 & 11.14 \\
\hline $\mathrm{T}_{4}(\mathrm{SB} @ 0.1 \%)$ & 25.04 & 22.21 & 20.11 & 18.67 & 16.02 & 14.02 & 11.88 \\
\hline $\mathrm{T}_{5}$ (SMS@ 0.05\%) & 25.04 & 22.91 & 19.21 & 17.89 & 15.97 & 13.61 & 10.89 \\
\hline $\mathrm{T}_{6}$ (SMS@0.1\%) & 24.99 & 22.31 & 20.17 & 18.91 & 16.03 & 15.04 & 10.98 \\
\hline $\mathrm{T}_{7}$ (PS@ 0.05\%) & 24.94 & 21.92 & 18.92 & 17.92 & 15.54 & 13.77 & 9.89 \\
\hline $\mathrm{T}_{8}(\mathrm{PS} @ 0.1 \%)$ & 25.04 & 23.17 & 20.71 & 18.83 & 16.02 & 14.78 & 10.87 \\
\hline $\mathrm{T}_{9}(\mathrm{SB}+\mathrm{PS} @ 0.05 \%$ each$)$ & 24.93 & 23.26 & 20.81 & 18.33 & 15.93 & 13.91 & 10.91 \\
\hline $\mathrm{T}_{10}(\mathrm{KMS}+\mathrm{PS} @ 0.05 \%$ each$)$ & 24.89 & 23.59 & 20.69 & 19.05 & 17.03 & 15.00 & 12.57 \\
\hline $\mathrm{T}_{11}$ (SMS+PS@ 0.05\% each) & 24.87 & 23.23 & 19.89 & 18.62 & 15.91 & 13.78 & 11.03 \\
\hline $\mathrm{T}_{12}$ (Refrigeration $\left(4 \pm 2{ }^{0} \mathrm{C}\right)$ & 25.05 & 23.89 & 21.67 & 19.24 & 17.16 & 15.02 & 12.88 \\
\hline $\mathrm{T}_{13}$ (Frozen storage $-20{ }^{\circ} \mathrm{C}$ ) & 25.15 & 23.91 & 21.87 & 19.84 & 17.43 & 16.23 & 13.02 \\
\hline SEm \pm & 0.53 & 0.52 & 0.52 & 0.37 & 0.36 & 0.26 & 0.26 \\
\hline C.D. $(\mathrm{P}=0.05)$ & NS & NS & 1.51 & 1.08 & 1.04 & 0.76 & 0.76 \\
\hline
\end{tabular}


Table.6 Effect of different preservatives and temperatures on reducing sugar (\%) content of mango pulp during storage

\begin{tabular}{|c|c|c|c|c|c|c|c|}
\hline \multirow[t]{2}{*}{ Treatments } & \multicolumn{7}{|c|}{ Storage duration (days) } \\
\hline & $\mathbf{0}$ & 15 & 30 & 45 & 60 & 75 & 90 \\
\hline $\mathrm{T}_{1}(\mathrm{KMS} @ 0.05 \%)$ & 9.79 & 9.86 & 9.99 & 10.08 & 10.11 & 10.24 & 10.32 \\
\hline $\mathrm{T}_{2}(\mathrm{KMS} @ 0.1 \%)$ & 9.84 & 10.31 & 10.67 & 10.93 & 11.04 & 11.46 & 11.78 \\
\hline $\mathrm{T}_{3}(\mathrm{SB} @ 0.05 \%)$ & 9.73 & 10.08 & 10.30 & 10.42 & 10.53 & 10.64 & 10.71 \\
\hline $\mathrm{T}_{4}(\mathrm{SB} @ 0.1 \%)$ & 9.79 & 10.25 & 10.42 & 10.53 & 10.64 & 11.20 & 11.37 \\
\hline T5 (SMS@ 0.05\%) & 9.81 & 9.89 & 9.97 & 10.18 & 10.26 & 10.34 & 10.41 \\
\hline $\mathrm{T}_{6}(\mathrm{SMS} @ 0.1 \%)$ & 9.73 & 9.86 & 9.97 & 10.13 & 10.37 & 10.68 & 10.74 \\
\hline $\mathrm{T}_{7}$ (PS@0.05\%) & 9.68 & 9.67 & 9.84 & 10.04 & 10.14 & 10.20 & 10.26 \\
\hline $\mathrm{T}_{8}(\mathrm{PS} @ 0.1 \%)$ & 9.68 & 9.74 & 9.97 & 10.08 & 10.18 & 10.28 & 10.36 \\
\hline $\mathrm{T}_{9}(\mathrm{SB}+\mathrm{PS} @ 0.05 \%$ each$)$ & 9.56 & 10.11 & 10.14 & 10.21 & 10.28 & 10.31 & 10.42 \\
\hline T10 $($ KMS+PS@ 0.05\% each) & 9.84 & 10.19 & 10.30 & 10.53 & 10.75 & 10.70 & 10.88 \\
\hline $\mathrm{T}_{11}$ (SMS+PS@ 0.05\% each) & 9.90 & 10.14 & 10.19 & 10.26 & 10.34 & 10.54 & 10.61 \\
\hline $\mathrm{T}_{12}$ (Refrigeration $\left(4 \pm 2{ }^{0} \mathrm{C}\right)$ & 9.84 & 10.52 & 10.73 & 10.93 & 11.01 & 11.54 & 11.94 \\
\hline $\mathrm{T}_{13}$ (Frozen storage $-20{ }^{\circ} \mathrm{C}$ ) & 9.90 & 10.54 & 11.01 & 11.34 & 11.58 & 11.84 & 12.04 \\
\hline $\mathrm{SEm} \pm$ & 0.15 & 0.23 & 0.28 & 0.11 & 0.12 & 0.10 & 0.07 \\
\hline C.D. $(\mathrm{P}=0.05)$ & NS & NS & NS & 0.33 & 0.34 & 0.30 & 0.20 \\
\hline
\end{tabular}

Table.7 Economics of different treatments of preservatives and temperature in mango pulp preservation

\begin{tabular}{lccccc}
\hline \multicolumn{1}{c}{ Preservation Methods } & $\begin{array}{c}\text { Cost } \\
\text { (Rs.) }\end{array}$ & $\begin{array}{c}\text { Gross return } \\
\text { (Rs.) }\end{array}$ & $\begin{array}{c}\text { Net return } \\
\text { (Rs.) }\end{array}$ & $\begin{array}{c}\text { B C } \\
\text { ratio }\end{array}$ & $\begin{array}{c}\text { Incremental } \\
\text { B:C over T }\end{array}$ \\
\hline $\mathrm{T}_{1}$ (KMS@ 0.05\%) & & & & & \\
& 2313 & 7500 & 5187 & 2.24 & 1.70 \\
$\mathrm{~T}_{2}$ (KMS@ 0.1\%) & 2326 & 9000 & 6674 & 2.87 & 2.18 \\
$\mathrm{~T}_{3}$ (SB@ 0.05\%) & 2314 & 7200 & 4886 & 2.11 & 1.60 \\
$\mathrm{~T}_{4}$ (SB@ 0.1\%) & 2328 & 8100 & 5772 & 2.48 & 1.89 \\
$\mathrm{~T}_{5}$ (SMS@ 0.05\%) & 2314 & 5550 & 3236 & 1.40 & 1.06 \\
$\mathrm{~T}_{6}$ (SMS@ 0.1\%) & 2328 & 6000 & 3672 & 1.58 & 1.20 \\
$\mathrm{~T}_{7}$ (PS@ 0.05\%) & 2342 & 5400 & 3058 & 1.31 & 1.00 \\
$\mathrm{~T}_{8}$ (PS@ 0.1\%) & 2384 & 5700 & 3316 & 1.39 & 1.08 \\
$\mathrm{~T}_{9}$ (SB+PS@ 0.05\% each) & 2356 & 7350 & 4994 & 2.12 & 1.63 \\
$\mathrm{~T}_{10}$ (KMS+PS@ 0.05\% each) & 2355 & 8250 & 5895 & 2.50 & 1.93 \\
$\mathrm{~T}_{11}$ (SMS+PS @ 0.05\% each) & 2356 & 7500 & 5144 & 2.18 & 1.68 \\
$\mathrm{~T}_{12}$ (Refrigeration $\left(4 \pm 2^{\circ} \mathrm{C}\right.$ ) & 2750 & 9000 & 6250 & 2.27 & 2.04 \\
$\mathrm{~T}_{13}$ (Frozen storage -20 ${ }^{\circ} \mathrm{C}$ ) & 2750 & 9600 & 6850 & 2.49 & 2.24 \\
\hline
\end{tabular}


In $\mathrm{pH}$ vice versa value was observed from acidity i.e., maximum in treatment $\mathrm{T}_{13}(3.85)$ and minimum in $\mathrm{T}_{7}$ (2.86) treatment. The increment in acidity of preserved mango pulp during storage period was due to formation of organic acids by degradation of ascorbic acids (Bal et al., 2014) and decrease in $\mathrm{pH}$ might be due to the formation of free acids and pectin hydrolysis (Ahmad et al., 2000).

\section{Ascorbic acid}

Ascorbic acid content of mango pulp was decreased with advancement of storage period (Table 5). The maximum ascorbic acid content was retained in treatment $\mathrm{T}_{13}(13.02$ $\left.\mathrm{mg} 100 \mathrm{~g}^{-1}\right)$ followed by $\mathrm{T}_{12}\left(12.88 \mathrm{mg} 100 \mathrm{~g}^{-1}\right)$ and minimum in treatment $\mathrm{T}_{7}(9.89 \mathrm{mg} 100$ $\left.\mathrm{g}^{-1}\right)$ at the end of storage period $\left(90^{\text {th }}\right.$ days $)$. Decrease in ascorbic acid content was due to the oxidation of ascorbic acid to de hydro ascorbic acid and then further degraded to 2, 3-diketo-gluconic acid by the action of ascorbic acid oxidase enzyme.

\section{Reducing sugar}

Table explicated that increment in reducing sugar with the advancement of storage period in all the treatments (Table 6), maximum reducing sugar content was recorded from treatment $\mathrm{T}_{13}$ (12.04 per cent) followed by $\mathrm{T}_{2}$ (11.94 per cent) and minimum in treatment $\mathrm{T}_{7}$ (10.26 per cent) at the end of the storage. It might be due to breakdown of some of the hemicelluloses and other saccharides into simple soluble sugars. The present study supported by the findings of Tandon and Kalra (1984) in guava pulp and Desai et al., (2012) in mango pulp.

\section{Economics}

From economic feasibility point of view the best treatment combination with maximum incremental B C ratio (2.24) and net return (₹
6850) was found to be $\mathrm{T}_{13}$ (Frozen storage $20^{\circ} \mathrm{C}$ ), however maximum B:C (2.87) was found with $\mathrm{T}_{2}$ (Table 7).

In conclusion, thus, mango pulp preservation under low temperature $\left(-20^{\circ} \mathrm{C}\right)$ gave superior quality and physico-chemical stability during 90 days of storage, however from economical analysis, preserving the pulp with KMS was best but frozen storage at $-20^{0} \mathrm{C}$ was found best from health point of view as compared to the chemical preservatives and may be recommended for the storage of mango pulp.

\section{References}

A.O.A.C. 1995. Official Methods of Analysis of Association of Official Analytical Chemists, Washington, D.C., 16: 37.

Ahmad, I., Khan, R. and Muhammad, A. 2000. Effect of added sugar at various concentrations on the storage stability of guava pulp. Sarhad J. Agri., 16(1): 89-93.

Akhtar, S., Riaz, M., Ahmad, A. and Nisar, A. 2010. Physio-chemical, microbiological and sensory stability of chemically preserved mango pulp. Pak. J. Botany, 42(2): 853-861.

Bal, L.M., Ahmad, T., Senapati, A.K. and Pandit, P.S. 2014. Evaluation of quality attribute during storage of guava nectar $c v$. Lalit from different pulp and TSS ratio. J. Food Processing and Technol., 5(5): 329-334.

Chandra, T. and Gehlot, R. 2006. Utilization of bael (Aegle marmelos Correa.) for preparation and preservation of pulp. Res. Crops, 7: 887-890.

Dubois, M., Gilles, K., Hamitton, J.K., Robers, P. A. and Smith, F. 1951. A colorimetric method for determination of sugar. Nature, 16: 167.

Fisher, R.A. 1950. Statistical Methods for Research Works. Oliver and Boyd. Edinburgh. 
Hussain, S., Rehman, S., Randhawa, M.A. and Iqbal, M. 2003. Studies on physiochemical, microbiological and sensory evaluation of mango pulp storage with chemical preservatives. J. Res. Sci., 14(1): 01-09.

Jain, P.K. and Nema, P.K. 2007. Processing of pulp of various cultivars of guava (Psidium guajava L.) for leather production. Agri. Engineering Int. CIGR J., 9: 1-9.

Miller, G.L. 1959. Determination of reducing sugar using 3, 5, Dinitrosalyclic acid. Anal. Chem., 31: 426-428.

Ramteke, R.S., Vijay Lakshmi, M.R. and Eipeson, W.E. 1999. Processing and value addition to mangoes. Indian Food Industry, 18: 155-163.

Saxena, M., Bhattacharya, S. and Malhotra, S.K. 2015. Horticultural statistics at a glance 2015. Oxford university press, New Delhi, p.186.

Sivakumar, D., Jiang, Y. and Yahia, E.M. 2011. Maintaining mango (Mangifera indica L.) fruit quality during the export chain. Food Res. Int., 44(5): 1254-1263. Tandon, D.K., Kumar, S., Dixit, A. and Shukla, D.K. 2010. Improvement in quality of beverages prepared from Rumani mango blended with Dashehari and Mallika. Indian J. Horticulture. 67(3): 376-380.

Tharanathan, R.N., Yashoda, H. M., and Prabha, T. N. 2006. Mango (Mangifera indica L.), The king of fruits-An overview. Food Reviews Int., 22(2): 6265.

Yadav, S.K., Sarolia, D.K., Pilania, S., Meena, H.R. and Mahawer, L.N. 2017. Studies on keeping quality of preserved guava pulp during storage. Int. J. Curr. Microbiol. Appl. Sci., 6(3): 1235-1242.

Yahia, E.M. and Ornelas-Paz, J. 2010. Chemistry, stability and biological actions of carotenoids. In: Fruits and vegetable phytochemicals: Chemistry, Nutritional Value and Stability (Eds de la Rosa, L.A., Alveraz-Parrilla, E. and Gonzalez-Aguilar, G.A.), pp. 177-222. Willey-Blackwell Publishing.

\section{How to cite this article:}

Manisha Jangir, Virendra Singh, Shalini Pilania, K.B. Shukla and Mahawer, L.N. 2017. Effect of Preservatives and Temperature on Physico-Chemical Properties and Economics of Mango (Mallika) Pulp. Int.J.Curr.Microbiol.App.Sci. 6(7): 2431-2438. doi: https://doi.org/10.20546/ijcmas.2017.607.288 